\title{
Relationship among dietary estimates of net endogenous acid production, bone mineral density and biochemical markers of bone turnover in an Iranian general population
}

\author{
Alireza Rahbar ${ }^{\mathrm{a}, *}$, Bagher Larijani ${ }^{\mathrm{b}}$, Iraj Nabipour ${ }^{\mathrm{c}}$, Mohamad Mehdi Mohamadi $^{\mathrm{c}}$, \\ Kamran Mirzaee ${ }^{c}$, Zahra Amiri $^{\mathrm{c}}$ \\ a Department of Nutrition, The Persian Gulf Tropical Medicine Research Center, Bushehr University of Medical Sciences, Bushehr, Islamic Republic of Iran \\ ${ }^{\mathrm{b}}$ Endocrinology and Metabolism Research Center, Tehran University of Medical Science, Islamic Republic of Iran \\ c Department of Cardiovascular Diseases, The Persian Gulf Tropical Medicine Research Center, Bushehr University of Medical Sciences, Bushehr, Islamic Republic of Iran
}

\section{A R T I C L E I N F O}

\section{Article history:}

Received 12 January 2009

Revised 17 March 2009

Accepted 14 July 2009

Available online 22 July 2009

Edited by: R. Baron

\section{Keywords:}

Net endogenous acid production (NEAP)

Dietary potassium

Bone mineral density

Bone turnover markers

Osteoporosis

Human

\begin{abstract}
A B S T R A C T
Chronic, low-grade metabolic acidosis due to Western diets may be a risk factor for osteoporosis. The severity can be determined in part by net endogenous acid production (NEAP). In a population-based study, a total of 1028 healthy men and women aged $20-72$ years were evaluated for dietary intakes and NEAP estimates with a validated food frequency questionnaire. Dual-energy X-ray absorptiometry (DXA) was used to determine BMD of the lumbar spine (L2-L4), distal third of radius, and proximal femur. Serum CrossLaps, degradation products of the C-terminal telopeptides of type I collagen, and osteocalcin were measured by highly specific ELISA methods. Lower estimates of energy-adjusted rates of NEAP were associated with greater femoral neck $\operatorname{BMD}(p=0.01)$ in premenopausal women and with greater BMDs at the distal radius $(p=0.001)$ and lumbar spine $(p=0.04)$ in postmenopausal women. Compared with women in the highest quartile of the estimates of the energy-adjusted rates of NEAP, pre- and postmenopausal women in the lowest quartile had significantly greater means of osteocalcin $[9.12(\mathrm{SD} \pm 1.62)$ vs. $5.24(\mathrm{SD} \pm 1.41) \mathrm{ng} / \mathrm{ml}, p=0.02$ and 11.74 $(\mathrm{SD} \pm 1.69)$ vs. $7.79(\mathrm{SD} \pm 2.63) \mathrm{ng} / \mathrm{ml}, p=0.002$, respectively]. Analysis by quartiles of the estimates of energy-adjusted rates of NEAP did not reveal a relationship between BMD and bone turnover markers in men. In conclusion, we found that a high energy-adjusted rate of NEAP was associated with a significantly lower BMD in women but not in men and the energy-adjusted rate of NEAP had a negative relationship with bone formation.
\end{abstract}

(c) 2009 Elsevier Inc. All rights reserved.

\section{Introduction}

Bone tissue plays a significant role in the control of the acid-base equilibrium of blood and extracellular fluid. In fact, even a slight degree of metabolic acidosis represents a strong stimulus for osteoclastic activity, and inhibits osteoblastic activity; on the other hand, metabolic alkalosis suppresses osteoclastic activity. This role of bone in acid-base regulation is likely to impact the maintenance of mineral stores and, in the long term, of bone mass [1].

One characteristic of the contemporary human diet for which no quantitative comparison has been made with the inferred ancestral preagricultural diet is the imbalance of nutrient precursors of hydrogen and bicarbonate ions, resulting in the body's net production of noncarbonic acid, ranging over an order of magnitude from 10 to $150 \mathrm{mEq} / \mathrm{d}$ among diets [2]. In other words, consumption of a normal

\footnotetext{
* Corresponding author. Deputy for Research, Bushehr University of Medical Sciences, Moallem Street, Bushehr, P. O. Box-3631, Islamic Republic of Iran. Fax: +98 7712528.
}

E-mail address: rahbar_alireza@yahoo.com (A. Rahbar).
Western diet is associated with chronic, low-grade metabolic acidosis [3], because metabolism of the Western diet releases noncarbonic acids into the systemic circulation (e.g., sulfuric acid from metabolism of protein) in amounts that exceed the amounts of base released concomitantly (e.g., bicarbonate from combustion of organic acid salts of potassium in vegetable foods) [4].

This persisting, albeit low-grade, acidosis and the relentless operation of responding homeostatic mechanisms may be an important contributional factor in numerous diseases such as nephrolithiasis, ageassociated reduction in lean body mass, and osteoporosis [5].

In vitro studies support the effect of acid-base balance on bone health; while acidosis inhibits osteoblasts and stimulates osteoclasts [6], alkalosis increases blood $\mathrm{pH}$, thereby shifting the balance in favor of the osteoblasts [7]. Mild acidosis also increases the activity of cathepsin $\mathrm{K}$, a metallo-protease secreted by osteoclasts for bonematrix resorption [8]. Even very small $\mathrm{pH}$ changes (as little as 0.05 ) result in a doubling or halving of resorption-pit formation in cultured osteoclasts [9]. Long-term acidosis also reduces IGF-1, and increases prostaglandin E2 (PGE2), RANKL, and M-CSF-all of which increase protein catabolism, muscle wasting, and bone resorption [10]. 
Supplementation of postmenopausal women with potassium bicarbonate has been shown to neutralize endogenous acid, reduce urinary calcium excretion, and alter bone remodeling in such a way as to improve calcium balance, at least over the short term [11,12].

In large population studies, measurement of the acid-base balance is not practical, but algorithms based on the ratio of protein to potassium have been used to estimate net endogenous (noncarbonic) acid production (NEAP) [4]. Frassetto et al. [4] proposed a simple algorithm to determine NEAP by measuring the acidifying effect of protein (via sulfate excretion) and the alkalizing effect of potassium (via provision of salts of weak organic acids).

Although indirect evidence is available of the effect of metabolic acidosis on bone from in vivo intervention studies and in vitro experiments, studies of bone density in general populations are limited -3 in women, 2 in children, and 1 in men and women [13-18].

New et al. [13] examined, for the first time, the association between NEAP and indexes of bone health by using a populationbased cohort of premenopausal and perimenopausal women. The results indicate that diets with a lower protein content but higher potassium content (i.e., lower acidity or high alkalinity) are associated with greater bone mass and a tendency to resorb less bone. The European Prospective Investigation into Cancer and Nutrition-Norfolk (EPIC-Norfolk) study was conducted in men and women, in which the relationship between a measure of dietary acid-base load, potential renal acid load (PRAL), and calcaneal broadband ultrasound attenuation (BUA) was estimated [18].

The purpose of the present cross-sectional study was to examine the relationship of net endogenous (noncarbonic) acid production (NEAP) and bone mineral density in an Iranian general population of women and men aged 20-72 years. Second, we investigated whether a relationship exists between NEAP and bone turnover markers in women and men.

\section{Materials and methods}

This study was performed as part of a larger epidemiological study, the Iranian Multicentral Osteoporosis Study (IMOS), in which 6000 normal subjects were randomly selected from five major cities throughout Iran. Details of the study can be found elsewhere [19]. Bushehr port (the center of Bushehr Province, which has the longest coastline on the Persian Gulf) was one of five cities that participated in the IMOS. The study goal was to recruit 1028 men and women, aged 20-72 years, in each age decade in every city. Participants were randomly selected from 13 geographical clusters in Bushehr port.

All participants were community-dwelling, and ambulatory. Publicity concerning the study appeared in local newspapers and on TV. The selected subjects were informed about the study through a letter given door-to-door by the survey groups of the IMOS. After a primary education about osteoporosis and its associated risk factors, the potential subjects were invited to participate in the study in a fasting state the following morning at the Persian Gulf Health Research Center at the Bushehr University of Medical Science.

All subjects answered a detailed questionnaire concerning covering demographic and behavioral information as well as medical history of conditions that could influence bone mass and metabolism. The women were also asked about medical history such as diabetes mellitus, inflammatory bowel diseases, epilepsy, chronic liver or renal diseases, cancer and self-reported hip or lumbar fractures. In addition, information was collected on reproductive history, including age at menarche and menopause, parity, use of postmenopausal hormone replacement therapy, and calcium and/or vitamin D supplementations. We considered women to be postmenopausal when they had had a hysterectomy or both ovaries removed or were aged 40 years and older and had not had a period for 12 months.
Height and weight were measured using a stadiometer. Heavy outer garments and shoes were removed before height and weight were measured.

\section{Measurements of bone indices}

Bone mineral density (BMD) was determined for the lumbar spine (L2-L4), the distal forearm, and the proximal femur (neck) using dual-energy X-ray absorptiometry (DXA) on the Osteocore II bone densitometer (Osteocore II Osteodensitometer, Medilink, France). The same operator tested all the women during the study to eliminate operator discrepancies. Duplicate measurements were obtained from 30 patients who agreed to undergo a repeat assessment on the same day, and precision errors were calculated using the root mean square method. The coefficients of variation (precision) of the measurements of the lumbar spine and femoral neck were $0.8 \%$ and $1.6 \%$, respectively.

The Serum CrossLaps ELISA (Nordic Bioscience Diagnostics A/S, Herlev, Denmark) was used for the quantification of the degradation products of the C-terminal telopeptides of type I collagen in sera. The intraassay CVs for the low $(0.242 \mathrm{ng} / \mathrm{ml})$, medium $(0.375 \mathrm{ng} / \mathrm{ml})$, and high $(0.476 \mathrm{ng} / \mathrm{ml})$ values were $5.4 \%, 5.0 \%$, and $5.1 \%$, respectively.

The N-MID Osteocalcin ELISA (Nordic Bioscience Diagnostics A/S, Herlev, Denmark) was used for the quantitative measurement of osteocalcin in sera. The intraassay CVs for the low $(7.0 \mathrm{ng} / \mathrm{ml})$, medium $(21.8 \mathrm{ng} / \mathrm{ml})$, and high $(43.2 \mathrm{ng} / \mathrm{ml})$ values were $3.4 \%, 2.0 \%$, and $2.4 \%$, respectively.

\section{Current dietary intake and estimation of NEAP}

Current dietary calcium intake and estimation of NEAP information was obtained through an interview conducted by trained dietitians. We determined the portion size and annual, monthly, and weekly frequency of intake using a food frequency questionnaire (FFQ). Reproducibility and relative validity of the 54-item FFQ were tested during the recruitment in the IMOS cohort by the Iranian Nutrition Institute $[19,20]$. The total calcium intake was estimated based on the same FFQ. Intakes of calcium, protein, and potassium were adjusted for total energy intake by normalizing all the intakes to the mean energy intake (residual method) in each group [21].

NEAP was estimated by examining the ratio of protein to potassium intake normalized to a diet of mean calories. The simple algorithm proposed by Frassetto et al. [4] was then applied.

$\mathrm{NEAP}=[62.1 \times$ protein $/ \operatorname{potassium}(\mathrm{mEq})]-17.9$

Each food and beverage was analyzed for content of energy and the other nutrients using NUNTRITIONIST III software (version 7.0; NSquared Computing, Salem, OR), which was designed for Iranian foods.

\section{Statistical analysis}

The distribution of variables was studied using probability plots and the Shapiro-Wilks test. We found that log transformation of Cross Laps and osteocalcin gave a better fit to a Gaussian distribution. The data for Cross Laps and osteocalcin were defined as the geometric mean (SD).

Group means were compared using the two-tailed non-paired Student's t-test. The relationship between the estimates of the energy-adjusted rate of NEAP or potassium intake and BMD and markers of bone turnover was also determined using analysis of covariance. Adjustments for age, weight, height, and postmenopausal years (for postmenopausal women) were made. Simple linear regression analysis was employed to study the relationships among the estimates of the energy-adjusted rate of NEAP or potassium intake, BMD measurements, and bone turnover markers. In addition, 
subjects were also divided into quartiles of the estimates of the energy-adjusted rate of NEAP or potassium intake and compared in the highest and lowest quartiles. With a calculated sample size of 1028 persons, the study will have a power of $80 \%$ to yield a statistically significant result. A value of $p<0.05$ was accepted as significant.

Statistical analysis was performed with an IBM computer using the SPSS 9.05 statistical software package (SPSS Inc., Chicago, IL).

\section{Results}

A total of 1028 (44.8\% males, 55.2\% females) subjects were evaluated. The response rate of individuals was $63 \%$. The mean ( \pm SD) age of the subjects was $42.5( \pm 13.4)$ years. Of the studied population, $24.1 \%$ was between 20 and 30 years, $20.9 \%$ between 31 and 40 years, $24.6 \%$ between 41 and 50 years, $19.0 \%$ between 51 and 60 , and $11.4 \%>60$ years. Table 1 shows the basic characteristics of the men and premenopausal and postmenopausal women. Significant differences between premenopausal and postmenopausal women were observed in bone mineral densities at different sites and biochemical bone markers (Table 1). There was no significant correlation between age and the estimates of NEAP but there was linear correlations between age and BMDs at different sites $(p<0.0001)$.

The dietary intakes of energy, protein, and potassium and estimates of NEAP (as protein: potassium intake) are shown in Table 1 for each group. The intakes of protein and potassium were well within the referenced nutrient intake. The mean $( \pm S D)$ estimate of the energy-adjusted rate of NEAP for all women and men was $100.57 \pm 87.69 \mathrm{mEq} / \mathrm{d}$.

Mean estimates of the energy-adjusted rate of NEAP quartiles (Q1Q4) were (59.98, 85.40, 105.26, and $188.18 \mathrm{mEq} / \mathrm{d}),(51.67,74.95$, 92.38 , and $141.35 \mathrm{mEq} / \mathrm{d})$ and $(36.23,62.17,94.41$, and $195.09 \mathrm{mEq} / \mathrm{d})$ for men, pre- and postmenopausal women.

In the age-adjusted analyses, there was a significant correlation between the estimates of the energy-adjusted rate of NEAP and BMDs at the distal third radius $(r=-0.13, p=0.04)$ and the femoral neck $(r=-0.1, p=0.02)$ in post- and premenopausal women, respectively.

No relationship was found between any skeletal sites and the estimates of the energy-adjusted rate of NEAP in the men.

The pre- and postmenopausal women were also divided into the highest and lowest quartiles of the estimates of the energy-adjusted rate of NEAP. Analysis by quartiles of the estimates of the energyadjusted rate of NEAP revealed a negative significant relationship with BMD at the femoral neck in premenopausal women $[0.964$ (SD \pm

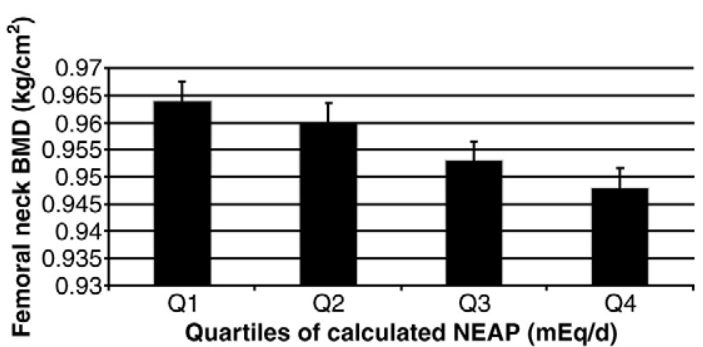

Fig. 1. Mean $( \pm S D)$ femoral neck bone mineral density (BMD) by quartiles $(Q)$ of net endogenous acid production (NEAP) in premenopausal women. Quartiles of NEAP (Q1-Q4) were 51.67, 74.95, 92.38, and $141.35 \mathrm{mEq} / \mathrm{d}$, respectively. Q1 was significantly different from Q4, $p=0.01$ (ANCOVA with LSD test, adjusted for age, weight and height).

$0.149)$ vs. $\left.0.947(\mathrm{SD} \pm 0.142) \mathrm{kg} / \mathrm{cm}^{2}, p=0.01\right]$ and with $\mathrm{BMDs}$ at the distal third radius $\left[0.501(S D \pm 0.110)\right.$ vs. $0.331(S D \pm 0.108) \mathrm{kg} / \mathrm{cm}^{2}$, $p=0.001]$ and lumbar spine $[0.898(\mathrm{SD} \pm 0.165)$ vs. $0.739(\mathrm{SD} \pm$ $0.164) \mathrm{kg} / \mathrm{cm}^{2}, p=0.04$ ] in postmenopausal women (Figs. 1-3). Analysis by quartiles of the estimates of the energy-adjusted rate of NEAP did not reveal a relationship with BMDs at the distal third radius [0.618 $(S D \pm 0.092)$ vs. $\left.0.748(S D \pm 0.112) \mathrm{kg} / \mathrm{cm}^{2}, p>0.05\right]$, and with lumbar spine $\left[0.995(S D \pm 0.177)\right.$ vs. $1.082(S D \pm 0.157) \mathrm{kg} / \mathrm{cm}^{2}$, $p>0.05]$ and with femoral neck $[0.977(\mathrm{SD} \pm 0.193)$ vs. $1.144 \pm$ $0.172 \mathrm{~kg} / \mathrm{cm}^{2}, p>0.05$ ] in men.

There was no significant age-adjusted linear correlation between the estimates of the energy-adjusted rate of NEAP and serum CrossLaps or osteocalcin in men and women.

But compared with the pre- and postmenopausal women in the highest quartile of the estimates of the energy-adjusted rate of NEAP, the pre- and postmenopausal women in the lowest quartile had significantly greater geometric means of osteocalcin $[9.12(S D \pm 1.62)$ vs. $5.24(\mathrm{SD} \pm 1.41) \mathrm{ng} / \mathrm{ml}, p=0.02$ and $11.74(\mathrm{SD} \pm 1.69)$ vs. 7.79 $(\mathrm{SD} \pm 2.63) \mathrm{ng} / \mathrm{ml}, p=0.002$, respectively]. Analysis by quartiles of the estimates of the energy-adjusted rate of NEAP did not reveal a relationship with serum osteocalcin $[10.00(S D \pm 1.64)$ vs. $12.30(S D \pm$ 1.69) $\mathrm{ng} / \mathrm{ml} p>0.05]$ and with serum Cross Laps [476.63 (SD \pm 1.77 ) vs. $467.73(\mathrm{SD} \pm 1.54) p>0.05$ ] in men.

The energy-adjusted potassium intake was significantly associated with a higher distal third radius BMD $(r=0.20, p=0.003)$ in postmenopausal women. In premenopausal women, this positive significant relationship was seen only between the estimates of the energy-adjusted potassium intake and femoral neck BMD $(r=0.10$,

Table 1

Basic characteristics of bone-related variables in an Iranian general population.

\begin{tabular}{|c|c|c|c|c|}
\hline & \multirow{2}{*}{$\begin{array}{l}\text { Men } \\
n=461\end{array}$} & \multicolumn{2}{|l|}{ Women } & \multirow{2}{*}{$\begin{array}{l}\text { Total } \\
n=567\end{array}$} \\
\hline & & $\begin{array}{l}\text { Premenopausal } \\
n=402\end{array}$ & $\begin{array}{l}\text { Postmenopausal } \\
n=165\end{array}$ & \\
\hline \multicolumn{5}{|l|}{ Characteristics } \\
\hline Age (year) & $44.9(13.7)$ & $37.63(8.53)^{a, b}$ & $56.43(6.63)^{\mathrm{a}}$ & $42.42(12.83)$ \\
\hline BMI $\left(\mathrm{kg} / \mathrm{m}^{2}\right)$ & $25.84(4.13)$ & $28.40(5.15)^{\mathrm{a}}$ & $28.48(4.78)^{a}$ & $28.09(5.04)^{a}$ \\
\hline Calcium intake (mg/d) & $850.2(757.0)$ & $750.6(433.0)^{a, b}$ & $704.0(419.0)^{\mathrm{a}}$ & $710.14(517.64)^{a}$ \\
\hline Protein $(\mathrm{gr} / \mathrm{d})$ & $76.49(20.08)$ & $68.66(18.05)^{a, b}$ & $54.57(15.62)^{\mathrm{a}}$ & $64.56(30.91)^{a}$ \\
\hline Potassium (mg/d) & $1668.7(871.8)$ & $1663.1(962.3)^{b}$ & $1305.1(661.5)^{a}$ & $1558.93(899.61)^{a}$ \\
\hline Energy $(\mathrm{kcal} / \mathrm{d})$ & $2351.5(1001.8)$ & $2000.4(879.9)^{a, b}$ & $1574.9(610.6)^{\mathrm{a}}$ & $1876.64(833.12)^{a}$ \\
\hline NEAP (mEq/d) & $109.69(112.66)$ & $91.55(46.33)^{a, b}$ & $97.04(82.80)^{\mathrm{a}}$ & $93.15(59.27)^{\mathrm{a}}$ \\
\hline \multicolumn{5}{|l|}{$B M D\left(\mathrm{~kg} / \mathrm{cm}^{2}\right)$} \\
\hline Radius & $0.528(0.084)$ & $0.566(0.099)^{\mathrm{b}}$ & $0.495(0.116)^{\mathrm{a}}$ & $0.548(0.103)$ \\
\hline Femoral neck & $0.949(0.166)$ & $0.962(0.151)^{\mathrm{b}}$ & $0.831(0.169)^{\mathrm{a}}$ & $0.924(0.165)$ \\
\hline Spine & $1.018(0.197)$ & $1.074(0.148)^{\mathrm{b}}$ & $0.899(0.166)^{a}$ & $1.015(0.178)$ \\
\hline Osteocalcin (ng/ml) & $11.22(1.62)$ & $8.91(1.62)^{a, b}$ & $11.48(1.69)$ & $9.77(1.65)^{a}$ \\
\hline CrossLaps (ng/ml) & $426.75(1.77)$ & $275.42(1.54)^{\mathrm{a}, \mathrm{b}}$ & $426.57(1.86)$ & $316.22(1.77)^{\mathrm{a}}$ \\
\hline
\end{tabular}

BMI: body mass index.

NEAP: net endogenous acid production.

a $p<0.01$ vs. men.

b $p<0.01$ vs. postmenopausal women. 


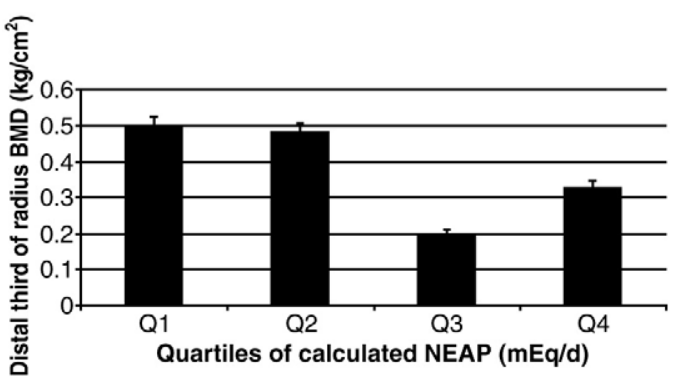

Fig. 2. Mean $( \pm S D)$ distal third of radius bone mineral density (BMD) by quartiles $(Q)$ of net endogenous acid production (NEAP) in postmenopausal women. Quartiles of NEAP (Q1-Q4) were 36.23, 62.17, 94.41, and $195.09 \mathrm{mEq} / \mathrm{d}$, respectively. Q1 was significantly different from Q3 and Q4, $p=0.001$ (ANCOVA with LSD test, adjusted for age, weight and height).

$p=0.02$ ). Analysis by quartiles of the energy-adjusted potassium intake revealed a positive significant relationship with BMD at the femoral neck in premenopausal women $[0.959(S D \pm 0.152)$ vs. 0.983 $\left.(\mathrm{SD} \pm 0.147) \mathrm{kg} / \mathrm{cm}^{2}, p=0.04\right]$ and with BMD at the distal third radius $\left[0.487(\mathrm{SD} \pm 0.108)\right.$ vs. $\left.0.761(\mathrm{SD} \pm 0.215) \mathrm{kg} / \mathrm{cm}^{2}, p=0.001\right]$ in postmenopausal women (Figs. 4,5 ). No relationship was found at any skeletal sites between BMD and the energy-adjusted potassium intake rate in men.

Analysis by quartiles of the energy-adjusted intake potassium intake did not reveal a relationship between serum Cross Laps and osteocalcin in men and premenopausal women. But in postmenopausal women, the highest quartile of the energy-adjusted potassium intake had significantly greater geometric means of osteocalcin [13.24 $(\mathrm{SD} \pm 1.79)$ vs. $7.87(\mathrm{SD} \pm 1.78) \mathrm{ng} / \mathrm{ml}, p=0.03$ ] (data not shown).

In the age-adjusted analyses, there was a significant correlation between the estimates of the energy-adjusted rate of NEAP and calcium and protein intakes. No relationship was found between any skeletal sites and calcium and protein intakes in men and pre- and postmenopausal women.

\section{Discussion}

In the present study, we found a significant negative relationship between a more acidic diet (higher estimates of energy-adjusted rates of NEAP) and BMD in women but not in men. Dietary potassium, an indicator of NEAP and fruit and vegetable intake, was associated with higher forearm and femoral BMDs in postmenopausal and premenopausal women, respectively. But there was no significant relationship between energy-adjusted rates of potassium intake and BMDs in men.

Our results agree with those by New et al. [13] that provided evidence of a positive link between a ratio of lower protein to higher potassium dietary intake (i.e., less dietary acid) and skeletal integrity in premenopausal and perimenopausal women. In the European Prospective Investigation into Cancer and Nutrition-Norfolk (EPIC-

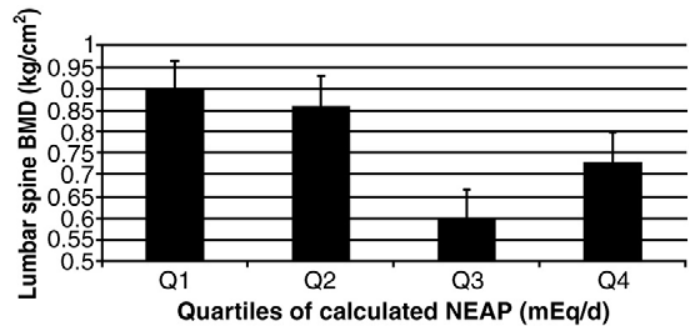

Fig. 3. Mean $( \pm S D)$ lumbar spine bone mineral density (BMD) by quartiles $(Q)$ of net endogenous acid production (NEAP) in postmenopausal women. Quartiles of NEAP (Q1-Q4) were 36.23, 62.17, 94.41, and $195.09 \mathrm{mEq} / \mathrm{d}$, respectively. Q1 was significantly different from Q3 and Q4, $p=0.04$ (ANCOVA with LSD test, adjusted for age, weight and height).

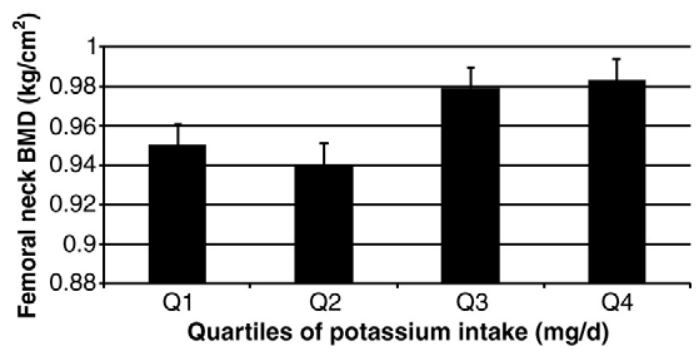

Fig. 4. Mean $( \pm S D)$ femoral neck bone mineral density (BMD) by quartiles $(Q)$ of dietary potassium intake in premenopausal women Quartiles of dietary potassium intake (Q1-Q4) were 1524.63, 3092.78, 4571.72 and $7990.12 \mathrm{mg} / \mathrm{d}$, respectively. Q1 was significantly different from Q4, $p=0.04$ (ANCOVA with LSD test, adjusted for age, weight and height).

Norfolk) study, a more acidic dietary intake (high PRAL) was significantly associated with lower calcaneal broadband ultrasound attenuation (BUA) in women but not in men; there was a difference of $\sim 2 \%$ in BUA between the highest and lowest quintiles of PRAL, independent of age, body mass index, smoking habit, physical activity, diagnosed osteoporosis, and history of fracture, and (in women) hormone replacement therapy [18].

Wynn et al. [15] assessed NEAP and quantitative bone ultrasound (QUS) in 401 very elderly Swiss ambulatory women. The authors found lower NEAP and higher potassium intake were correlated with higher BUA, which remained significant even after adjustment for age, BMI, and osteoporosis treatment.

Our data support the growing interest in the importance of acidbase homeostasis to bone health. It has been shown that when acid production is experimentally increased among healthy subjects, renal net acid excretion does not increase as much as acid production so that acid balances become positive. These positive imbalances are accompanied by equivalently negative charge balances that are the result of bone buffering of retained $\mathrm{H}+$ and loss of bone $\mathrm{Ca} 2+$ in the urine [22].

On a larger epidemiological scale, it is interesting to speculate on the effects of diet-induced acid loads. In the contemporary diet, bicarbonate production rates are disproportionately lower, thereby tipping the balance from net base to net acid production [2]. It is conceivable, although speculative, that the chronic acid load induces a state of chronic nitrogen wasting. This, together with the blunted acid excretion associated with ageing, could contribute to the progressive decrease in muscle and bone mass in older people [5]. Interestingly, Sebastian et al. have shown that neutralization of endogenous acid production (by administering potassium bicarbonate) in postmenopausal women results in calcium retention and decreased bone resorption markers. In addition, renal nitrogen excretion decreased significantly $[12,23]$.

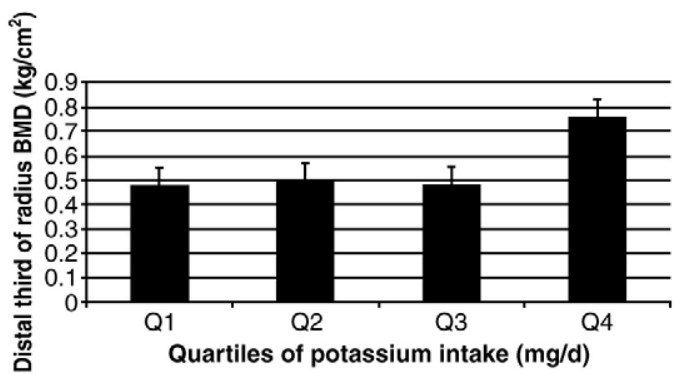

Fig. 5. Mean $( \pm S D)$ distal third of radius bone mineral density (BMD) by quartiles $(Q)$ of dietary potassium intake in postmenopausal women. Quartiles of dietary potassium intake (Q1-Q4) were 986.78, 1548.28, 2886.80 and $4536.60 \mathrm{mg} / \mathrm{d}$, respectively. Q1 was significantly different from Q4, $p=0.001$ (ANCOVA with LSD test, adjusted for age, weight and height). 
Therefore, avoiding high amounts of acid ash-forming food and increasing the intake of alkalizing food and mineral water rich in bicarbonate may have a favorable influence on the maintenance of bone mass.

We found no relationship between the estimates of the energyadjusted rate of NEAP and BMD at any site in men in this population. Only one study about the relationship between acidic dietary acidbase load and bone health in men has been published [18]. The researchers also found no relationship between dietary intake of PRAL and BUA in men. Why women are more sensitive than men to the effects of dietary acid load remains an open question.

In this study, postmenopausal and premenopausal women with the highest energy-adjusted potassium intake had significantly higher BMDs at the forearm and the femoral neck. The beneficial effects of dietary potassium on bone health are well established in the literature $[24,25]$. A higher BMD has been associated with a higher dietary potassium intake, along with other nutrients associated with fruit and vegetable intake [26].

It has been suggested that potassium, as an integral part of acidbase homeostasis, buffers the endogenous acids derived from the Western diet. In fact, bicarbonate is not present in foods and may be produced within host tissues as a consequence of the oxidation of organic anions (essentially $\mathrm{K}+$ malateor citrate salts). Potassium and organic anions, through $\mathrm{KHCO}$-generation or glutamine sparing, are very effective in neutralizing mineral acidity and favoring neutral or slightly alkaline urine $\mathrm{pH}[27]$.

We did not find a relationship between Cross Laps, as a bone resorption marker, and the estimates of the energy-adjusted rate of NEAP in men and women. A reduction in bone resorption markers was reported in potassium supplementation studies [12,28,29]. But there is only one population-based study in the literature. It showed that, for women around the time of menopause, NEAP was associated with increased concentrations of urinary bone resorption markers [14]. To our knowledge, the present study is the first large population-based study of net endogenous (noncarbonic) acid production (NEAP) and markers of bone turnover in men and premenopausal and postmenopausal women. The lack of a relationship between serum Cross Laps and NEAP in the present study may be due to the low sensitivity of serum Cross Laps in detecting the effects of physiologically normal limits of mild, low-grade acidosis in bone health.

Osteocalcin, a protein product of mature osteoblasts, is involved in the adequate development of bone matrix. Elevated serum osteocalcin concentrations indicate an increased rate of bone formation and turnover, but how osteocalcin may be influenced by menopause is unclear [30]. In the present study, the results for pre- and postmenopausal women showed that the energy-adjusted rate of NEAP was negatively associated with osteocalcin; this bone formation marker was significantly greater for women in the lowest quartile of the energy-adjusted rate of NEAP compared to those in the highest quartile. This finding demonstrated that the energy-adjusted rate of NEAP has a more prominent effect on bone formation compared to resorption in postmenopausal women. Sebastian et al. [12] showed that supplementation with potassium bicarbonate in postmenopausal women reduced urinary excretion of hydroxyproline, in association with an increase in the serum osteocalcin concentration. Thus, they concluded that the administration of potassium bicarbonate appeared to reduce the rate of bone resorption and increase the rate of bone formation.

There are several limitations to this population-based study. First, we did not measure sex hormone concentrations or other endocrine or paracrine factors that may alter local and systemic mild, low-grade metabolic acidosis, including local cytokines and OPG/RANKL ratio, all of which may have cumulative and interactive effects. These are of value in understanding the mechanisms by which chronic acidity influences bone integrity. Second, the cross-sectional associations limit the causal inferences that can be made, although there are biologically plausible mechanisms for the relationship between dietary acid load and bone metabolism. It is unlikely that persons with different bone measures across the normal range would change their dietary intake [18]. Third, the data on diet and NEAP were obtained by self-reported questionnaire (which could lead to possible over reporting on specific aspects of the diet, such as fruit and vegetable consumption). We did not measure in a subgroup the true NEAP estimate from 24-h urine collections.

In conclusion, we found that a high energy-adjusted rate of NEAP was associated with a significantly lower BMD in women but not in men and the energy-adjusted rate of NEAP had a negative association with bone formation. However, observational studies do not prove hypotheses and can only suggest the potential mechanisms of action. We now urgently need data from randomized controlled trials to determine for certain whether a potassium-rich, bicarbonate-rich diet or supplement is important to the skeleton [31]. Studies will also be needed to examine the effects on fracture rates in large population studies.

\section{Acknowledgments}

This study was supported in part by a grant from Joint Ministry of Health and Endocrinology and Metabolism Research Center, Tehran University of Medical Science and The Persian Gulf Tropical Medicine Research Center, Bushehr University of Medical Sciences, Bushehr, I.R. Iran (DP/20/18/3/1237,8/9/2005). We wish to thank Seyed Mojtaba Jafari, Zahra Sanjdeh and Shiva Mosadeghzadeh for coordinating the collection of samples and for laboratory management.

\section{References}

[1] Buclin T, Cosma M, Appenzeller M, Jacquet AF, Decosterd LA, Biollaz J, et al. Diet acid and alkalis influence calcium retention in bone. Osteoporosis Int 2001;12: 493-9.

[2] Sebastian A, Frassetto LA, Sellmeyer DE, Merriam RL, Morris Jr RC. Estimation of the net acid load of the diet of ancestral preagricultural Homo sapiens and their hominid ancestors. Am J Clin Nutr 2002;76(6):1308-16.

[3] Kurtz I, Maher T, Hulter HN, Schambelan M, Sebastian A. Effect of diet on plasma acid-base composition in normal humans. Kidney Int 1983;24:670-80.

[4] Frassetto L, Todd K, Morris Jr RC, Sebastian A. Estimation of net endogenous noncarbonic acid production in humans from dietary protein and potassium contents. Am J Clin Nutr 1998;67:576-83.

[5] Wiederkehr M, Krapf R. Metabolic and endocrine effects of metabolic acidosis in humans. Swiss Med Wkly 2001;131:127-32.

[6] Krieger NS, Sessler NE, Bushinsky DA. Acidosis inhibits osteoblastic and stimulates osteoclastic activity in vitro. Am J Physiol 1992;262:F442-8.

[7] Bushinsky DA. Metabolic alkalosis decreases bone calcium efflux by suppressing osteoclasts and stimulating osteoblasts. Am J Physiol 1996;271:F216-22.

[8] Brandao-Burch A. Mild acidosis upregulates mRNA for cathepsin K and Traf.6, increase OC activity, and decrease OB function. 30th European Symposium on Calcified Tissues. May 8-12, 2003.

[9] Arnett T. Regulation of bone cell function by acid-base balance. Proc Nutr Soc 2003;62:511-20.

[10] McCormick RK. Osteoporosis: integrating biomarkers and other diagnostic correlates into the management of bone fragility. Altern Med Rev 2007;12: $113-45$.

[11] Wood RJ. Potassium bicarbonate supplementation and calcium metabolism in postmenopausal women: are we barking up the wrong tree? Nutr Rev 1994;52: 278-80.

[12] Sebastian A, Harris ST, Ottaway JH, Todd KM, Morris Jr RC. Improved minera balance and skeletal metabolism in postmenopausal women treated with potassium bicarbonate. N Engl J Med 1994;330:1776-81.

[13] New SA, Macdonald HM, Campbell MK, et al. Lower estimates of net endogenous non-carbonic acid production are positively associated with indexes of bone health in premenopausal and perimenopausal women. Am J Clin Nutr 2004;79: 131-8.

[14] Macdonald HM, New SA, Fraser WD, Campbell MK, Reid DM. Low dietary potassium intakes and high dietary estimates of net endogenous acid production are associated with low bone mineral density in premenopausal women and increased markers of bone resorption in postmenopausal women. Am J Clin Nutr 2005;81:923-33.

[15] Wynn E, Lanham-New SA, Krieg MA, Whittamore DR, Burckhardt P. Low estimates of dietary acid load are positively associated with bone ultrasound in women older than 75 years of age with a lifetime fracture. J Nutr 2008;138:1349-54.

[16] Alexy U, Remer T, Manz F, Neu CM, Schoenau E. Long-term protein intake and dietary potential renal acid load are associated with bone modeling and 
remodeling at the proximal radius in healthy children. Am J Clin Nutr 2005;82: 1107-14.

[17] Chan RS, Woo J, Chan DC, Lo DH, Cheung CS. Bone mineral status and its relation with dietary estimates of net endogenous acid production in Hong Kong Chinese adolescents. Br J Nutr 2008;100:1283-90.

[18] Welch AA, Bingham SA, Reeve J, Khaw KT. More acidic dietary acid-base load is associated with reduced calcaneal broadband ultrasound attenuation in women but not in men: results from the EPIC-Norfolk cohort study. Am J Clin Nutr 2007;85:1134-41.

[19] Larijani B, Moayyeri A, Keshtkar AA, Hossein-Nezhad A, Soltani A, Bahrami A, et al Peak bone mass of Iranian population: the Iranian Multicenter Osteoporosis Study. J Clin Densitom 2006;9:367-74.

[20] Hashemipour S, Larijani B, Adibi H, Javadi E, Sedaghat M, Pajouhi M, et al. Vitamin $\mathrm{D}$ deficiency and causative factors in the population of Tehran. BMC Public Health 2004;4:38.

[21] Willett WC, Howe GR, Kushi LH. Adjustment for total energy intake in epidemiologic studies. Am J Clin Nutr 1997;65:1220s-8s.

[22] Lemann Jr J, Bushinsky DA, Hamm LL. Bone buffering of acid and base in humans. Am J Physiol, Renal Physiol 2003;285:F811-32.

[23] Frassetto L, Morris Jr RC, Sebastian A. Potassium bicarbonate reduces urinary nitrogen excretion in postmenopausal women. J Clin Endocrinol Metab 1997;82:254-9.
[24] Whiting SJ, Boyle JL, Thompson A, Mirwald RL, Faulkner RA. Dietary protein, phosphorus and potassium are beneficial to bone mineral density in adult men consuming adequate dietary calcium. J Am Coll Nutr 2002;21:402-9.

[25] Macdonald HM, New SA, Golden MH, Campbell MK, Reid DM. Nutritional associations with bone loss during the menopausal transition: evidence of a beneficial effect of calcium, alcohol, and fruit and vegetable nutrients and of a detrimental effect of fatty acids. Am J Clin Nutr 2004;79:155-65.

[26] Prentice A. Diet, nutrition and the prevention of osteoporosis. Public Health Nutr 2004; 7:227-43.

[27] Demigné C, Sabboh H, Rémésy C, Meneton P. Protective effects of high dietary potassium: nutritional and metabolic aspects. J Nutr 2004;134:2903-6.

[28] Sellmeyer DE, Schloetter M, Sebastian A. Potassium citrate prevents increased urine calcium excretion and bone resorption induced by a high sodium chloride diet. J Clin Endocrinol Metab 2002;87:2008-12.

[29] Marangella M, Di Stefano M, Casalis S, Berutti S, D'Amelio P, Isaia GC. Effects of potassium citrate supplementation on bone metabolism. Calcif Tissue Int 2004;74: 330-5.

[30] Calvo MS, Eyre DR, Gundberg CM. Molecular basis and clinical application of biological markers of bone turnover. Endocr Rev 1996;17:333-68.

[31] Lanham-New SA. The balance of bone health: tipping the scales in favor of potassium-rich, bicarbonate-rich foods. J Nutr 2008;138:172S-7S. 